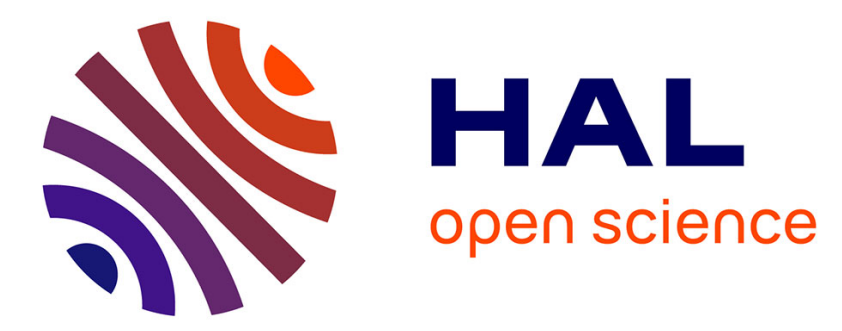

\title{
Vibronic spectra of protonated hydroxypyridines: contributions of prefulvenic and planar structures
}

R Lozada Garcia, N Nieuwjaer, Charles Desfrançois, F Lecomte, S D Leite, M Manil, M Broquier, G Grégoire

\section{- To cite this version:}

R Lozada Garcia, N Nieuwjaer, Charles Desfrançois, F Lecomte, S D Leite, et al.. Vibronic spectra of protonated hydroxypyridines: contributions of prefulvenic and planar structures. Physical Chemistry Chemical Physics, 2017, 19 (12), pp.8258-8268. 10.1039/C6CP08623C . hal-01494656

\section{HAL Id: hal-01494656 \\ https://sorbonne-paris-nord.hal.science/hal-01494656}

Submitted on 23 Mar 2017

HAL is a multi-disciplinary open access archive for the deposit and dissemination of scientific research documents, whether they are published or not. The documents may come from teaching and research institutions in France or abroad, or from public or private research centers.
L'archive ouverte pluridisciplinaire HAL, est destinée au dépôt et à la diffusion de documents scientifiques de niveau recherche, publiés ou non, émanant des établissements d'enseignement et de recherche français ou étrangers, des laboratoires publics ou privés. 


\title{
Vibronic spectra of protonated hydroxypyridines: contributions of prefulvenic and planar
} structures.

R. Lozada Garciaa ${ }^{a}$ N. Nieuwjaera, C. Desfrançois ${ }^{a}$, F. Lecomtea, S.D. Leite ${ }^{a}$, B. Manila, M. Broquier ${ }^{b, c}$, G. Grégoire ${ }^{b, c}$

a Laboratoire de Physique des Lasers, CNRS, Université Paris 13, Sorbonne Paris Cité, 93430 Villetaneuse, France.

${ }^{b}$ Institut des Sciences Moléculaires d'Orsay (ISMO), CNRS, Univ. Paris Sud, Université Paris-Saclay, F-91405 Orsay (France)

${ }^{c}$ Centre Laser de l'Université Paris-Sud (CLUPS/LUMAT), Univ. Paris-Sud, CNRS, IOGS, Université Paris-Saclay, F-91405 Orsay (France)

\begin{abstract}
Various hydroxypyridine derivatives are endogenous or synthetic photosensitizers which could contribute to solar radiation damages. The study of their excited states could lead to a better understanding of their action mechanisms. We present here the ultraviolet (UV) spectra of the protonated 2-, 3- and 4-hydroxypyridine. These spectra were obtained with an experimental device coupling an electrospray ion source with a cold quadrupole ion trap and a time of flight mass spectrometer. They display well resolved vibrational structures, with a clear influence of the position of the $\mathrm{OH}$ group. These results are interpreted with excited states calculations at the coupled cluster CC2 level.
\end{abstract}

\section{1 - Introduction}

Because photochemistry underlies the function of many biomolecules and can initiate DNA damage and thus cause disease, ${ }^{1,2}$ there is significant interest in the UV photochemistry of aromatic heterocycles relevant to biology. ${ }^{3-5}$ The deactivation of excited DNA molecules may involve local, "monomer-like » processes, ${ }^{2}$ which reinforces the importance of understanding the photochemistry of model molecules that mimic the molecular subunits comprising larger biologically relevant compounds. 2-, 3- and 4-hydroxypyridines are such model systems. Indeed, 2hydroxypyridine exhibits two stable tautomeric forms: the enol form and the keto one called 2- 
pyridone. These two tautomers are related by $\mathrm{H}$ atom transfer between $\mathrm{N}$ and $\mathrm{O}$ sites of the molecule and they are nitrogen heterocycles with the same $\mathrm{H}$-bonding sites as uracil and thymine. These similarities make the mixed 2-pyridone 2-hydroxypyridine dimer a relevant model compound for tautomeric DNA-base pair analogues. ${ }^{6}$ As a consequence, gas phase spectroscopy of the neutral 2-hydroxypyridine in molecular beams has been extensively documented over the last decades. ${ }^{7-11}$ Concerning 3-hydroxypyridine, its cation has been identified as a minimum phototoxic chromophore sufficient to effect skin cell sensitization ${ }^{12}$ and 3-hydroxypyridine derivatives, comprising a wide range of skin biomolecules, are capable of skin photo-oxidative damage. ${ }^{13}$ This molecule is then more than a model system, and the study of its excited states could lead to a better understanding of its action mechanisms.

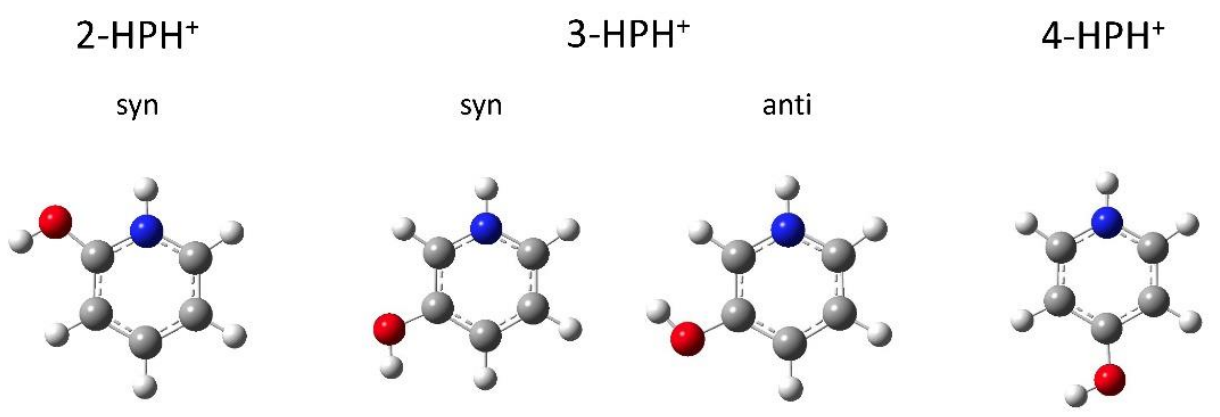

Chart 1: Structures of the three investigated protonated hydroxypyridines $\mathrm{HPH}^{+}$

We present here a systematic study of the influence of the position of the hydroxyl group in protonated hydroxypyridine molecules (see chart 1). Production and spectroscopic studies of protonated aromatic amino acids ${ }^{14-17}$ and of the protonated DNA/RNA bases ${ }^{18-20}$ and their homodimers $^{21}$ have merged recently and have been made possible by the coupling of an electrospray source with a cold ion trap. ${ }^{22}$ Well-resolved vibronic spectroscopy can thus be recorded and assigned through comparison with ab initio quantum mechanics calculations. Studying model systems gives insight into the photochemistry of protonated nitrogen heterocycles that in turn aids in understanding biologically relevant photochemistry. The simplest protonated aromatic molecule, benzene, and aromatic nitrogen heterocycle, pyridine, have been characterized at medium resolution only very recently. ${ }^{23,24}$ The electronic spectrum of protonated benzene is structure-less, which is in agreement with the large geometrical changes and the fast dynamic toward internal conversion predicted by ab-initio calculations. ${ }^{25}$ Concerning protonated pyridine, the spectra recorded in the photon energy range $37000-45000 \mathrm{~cm}^{-1}$ reveal a broad electronic band exhibiting vibronic resolved structure. The excited state structure is buckled in a prefulvenic geometry. This 
behavior is a familiar feature of many small aromatics ${ }^{26-28}$ and is also observed for neutral pyridine. ${ }^{29-32}$ Very recently, Broquier et al. have undertaken the investigation of protonated ortho, meta and para aminopyridine molecules ${ }^{33,34}$ and have shown that although protonated aminopyridines are rather simple aromatic molecules, their deactivation mechanisms are indeed quite complex. In the present study, the spectroscopy of the protonated 2-, 3- and 4hydroxypyridine $\left(\mathrm{HPH}^{+}\right)$is investigated across the photon energy range $33500-41000 \mathrm{~cm}^{-1}$ using an experimental device coupling an electrospray ion source with a cold quadrupolar ion trap and a time of flight mass spectrometer. We present the vibrationally resolved electronic spectra of cold $\mathrm{HPH}^{+}$along with dissociation kinetics through one- and two-color photofragmentation spectroscopy. Ultraviolet-ultraviolet hole-burning (UV-UV HB) spectroscopy was also used to discriminate conformational isomers in the case of the protonated meta 3-hydroxypyridine.

\section{2 - Experimental and computational methods}

2-, 3- and 4-hydroxypyridine were purchased from Sigma Aldrich and directly dissolved in water/methanol mixtures (50/50 by volume) at $100 \mu \mathrm{M}$ and together with few droplets of acetic acid. The electronic spectra were obtained via photo-fragmentation spectroscopy in a cryogenic cold quadrupole ion trap QIT (Paul trap from Jordan TOF Products, Inc.) installed at the CLUPS facility in Orsay. The setup is similar to the one developed by X. B. Wang et al. ${ }^{35}$ and has already been described elsewhere. ${ }^{36,37}$ The protonated species are produced from an Electrospray lon Source (ESI). They pass through a $10 \mathrm{~Hz}$ pulsed octopole ion guide that produces ion packets with a duration time between $500 \mathrm{~ns}$ and $1 \mu \mathrm{s}$, and are then trapped in the QIT which is mounted on a cold head of a compressed helium cryostat that maintains the temperature around 10-15 K. A mass gate located before the entrance of the trap allows for mass-selecting the parent ion. The photodissociation laser is triggered after few tens of millisecond when thermalization of the ions through collisions with helium buffer gas is reached, and all ionic fragments and parent molecules are then extracted and accelerated for mass-analysis in a linear time-of-flight mass spectrometer. The photodissociation laser is either a picosecond Optical Parametric Amplifier (OPA), with a spectral resolution of $8 \mathrm{~cm}^{-1}$, or a nanosecond dye laser (Quantel TDL 90), which resolution is about $0.2 \mathrm{~cm}^{-1}$.

For the fragmentation kinetics measurements, the fragmentation time is recorded by changing the delay between the laser and the extraction pulse ejecting the ions from the ion trap to the time-offlight. The time resolution, on the order of $80 \mathrm{~ns}$, is limited by the rise time of the high voltage pulser. 
UV-UV hole burning (HB) spectroscopy (using two dye lasers) has been performed in order to reveal the possible distinct isomers that are present in the trap after the low temperature cooling process. We have used the tickle method initially proposed by Kang et al., ${ }^{38}$ which consists of applying on one cap of the QIT an auxiliary RF whose frequency is tuned to selectively eject a given ionic fragment. By doing that, the chosen ionic fragment of a first UV laser (burn), set on a given vibronic band, is ejected from the trap, which allows recording the signal induced by a second UV laser (probe) scan, over the whole spectral range, without background signal. The tickle RF voltage is kept below $1 \mathrm{~V}$ and sent for $10 \mathrm{~ms}$ after the excitation from the burn laser. In order to increase the HB signal-to-noise ratio, we run the burn laser at $5 \mathrm{~Hz}$ while keeping the probe at $10 \mathrm{~Hz}$ and perform the experiment using the modified active baseline subtraction methods (ABS). ${ }^{39,40}$

\section{Computational methods}

$A b$ initio calculations have been performed with the TURBOMOLE program package $(v 6.2)^{41}$ making use of the resolution-of-the-identity (RI) approximation for the evaluation of the electron-repulsion integrals. ${ }^{42}$ The equilibrium geometries of protonated 2-, 3- and 4-hydroxypyridines in their ground electronic $\left(\mathrm{S}_{0}\right)$ and excited states have been determined at the CC2 level with the correlationconsistent polarized valence double- $\zeta$ aug-cc-pVDZ basis set augmented with diffuse functions. ${ }^{43}$ The vibrational modes of the ground and the first excited states have been calculated at the same level, and the Franck-Condon analysis has been performed using PGOPHER software. ${ }^{44}$

\section{Isomers}

Hydroxypyridines are protonated at the pyridinic nitrogen and can adopt two conformations according to the position of the oxygen lone pair as compared to the position of the proton. The conformer with the lone pair oriented towards the proton will be denoted syn, and the conformer with a rotation of $\pi$ around the $\mathrm{C}-\mathrm{O}$ bond will be denoted anti. For protonated 2-hydroxypyridine, the anti conformer is likely to be unstable due to the repulsion between the proton bound to nitrogen and the hydroxyl hydrogen. For the protonated 4-hydroxypyridine, the two isomers are identical due to the NCO symmetry axis. Only in the case of protonated 3-hydroxypyridine, the two isomers can coexist and are energetically close. As compared to the neutral molecule, the protonated hydroxypyridine can hardly display tautomerization because the keto form would correspond to a positively charge doubly hydrogenated nitrogen atom with a $\mathrm{sp}^{3}$ tetrahedral molecular orbital structure calculated $1.7 \mathrm{eV}$ higher than the initial $\mathrm{sp}^{2}$ hybridization. 


\section{3 - Results}

\section{Protonated 2-Hydroxypyridine : 2-HPH}

Figure 1a shows the vibrationally-resolved UV photofragmentation spectrum obtained with the ps OPA laser on a large spectral domain, from $34500 \mathrm{~cm}^{-1}$ up to $2000 \mathrm{~cm}^{-1}$ above. The UV excitation of 2- $\mathrm{HPH}^{+}\left(\mathrm{m} / \mathrm{z}\right.$ 96) results in the dissociation into $\mathrm{m} / \mathrm{z} 78$ ions, which corresponds to the loss of $\mathrm{H}_{2} \mathrm{O}$. This fragment, which is also the main dissociation channel in CID, is produced immediately with a kinetics of formation shorter than the time resolution of the experiment defined by the rising time of 80 ns of the extraction pulse from the QIT.

The vibronic spectrum of $2-\mathrm{HPH}^{+}$is composed of intense and sharp bands, the first one being observed at $35435 \pm 5 \mathrm{~cm}^{-1}$, while low intensity transitions are barely seen in the red of this intense band. The higher resolution spectrum obtained with the dye laser is reported in Fig. $1 \mathrm{~b}$ and confirms that the first weak vibronic band is indeed recorded at $34806 \pm 5 \mathrm{~cm}^{-1}$, i.e. about $-630 \mathrm{~cm}^{-1}$ from the intense band. These weak transitions cannot be assigned to hot bands because they are too far in the red from the intense band. In the low frequency region, there is a vibrational progression of $+82 \mathrm{~cm}^{-1}$ built from the first transition at $34806 \mathrm{~cm}^{-1}$ with at least 2 quanta with increasing intensity. This suggests a geometry distortion in the excited state along a low frequency mode, leading to a weak band origin. The low signal makes a firm assignment of all the observed transitions difficult. We can nevertheless recover the same vibrational progression built from a band at $390 \mathrm{~cm}^{-1}$ above the first transition. In overall, the vibronic spectrum of $2-\mathrm{HPH}^{+}$indeed closely resembles to the one recorded for protonated 2-aminopyridine. ${ }^{33}$ As it will discussed below, these two sets of intense and weak transitions will be assigned to two distinct minima in the excited state of $2-\mathrm{HPH}^{+}$, as observed for protonated 2-aminopyridine. The band origin of $2-\mathrm{HPH}^{+}$is red-shifted by more than $1300 \mathrm{~cm}^{-1}$ as compared to neutral 2-hydroxypyridine. . $^{7,-10}$ 

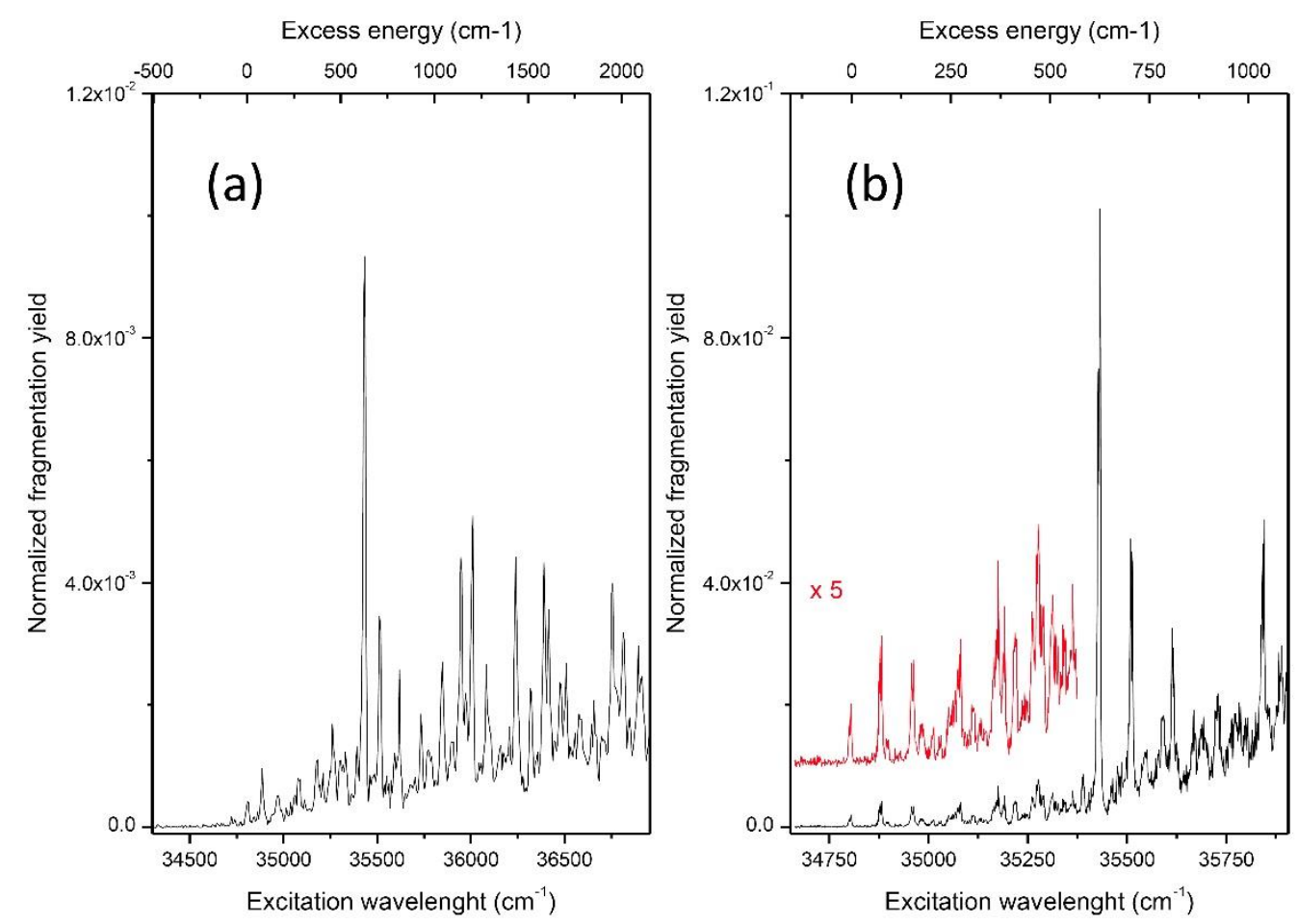

Figure 1. Photofragmentation spectrum of protonated $2-\mathrm{HPH}^{+}$recorded on the $\mathrm{m} / \mathrm{z} 78$ ionic fragment. (a) on the left, the spectrum is obtained over a large spectral range with the ps OPA laser ( $8 \mathrm{~cm}^{-1}$ resolution). (b) on the right, low frequency region obtained with the dye laser $\left(0.2 \mathrm{~cm}^{-1}\right.$ resolution).

\section{Protonated 3-Hydroxypyridine : 3-HPH ${ }^{+}$}

The vibrationally resolved vibronic spectrum of $3-\mathrm{HPH}^{+}$, shown in Figure $2 \mathrm{a}$, is obtained with the OPA laser from $33500 \mathrm{~cm}^{-1}$ up to $3000 \mathrm{~cm}^{-1}$ above. The UV-induced fragmentation produces mainly two fragment ions that are also observed through CID: $\mathrm{m} / \mathrm{z} 68$, which is the main fragment and corresponds to the loss of $\mathrm{CO}$, and $\mathrm{m} / \mathrm{z} 78$, which is ten times less intense and corresponds to the loss of $\mathrm{H}_{2} \mathrm{O}$, as observed for 2- $\mathrm{HPH}^{+}$. A fragmentation kinetics of $210 \mu$ s for the two fragmentation channels has been measured and is consistent with a dissociation in the ground state following internal conversion. As for $2-\mathrm{HPH}^{+}$, the vibronic spectrum is composed of a set of intense transitions starting a $34357 \pm 5 \mathrm{~cm}^{-1}$ along with low intensity bands further in the red. The higher resolution spectrum recorded with the dye laser (Fig. 2b) allows asserting the position of the first weak transition at $33814 \mathrm{~cm}^{-1}$, i.e. about $540 \mathrm{~cm}^{-1}$ in the red of the intense band. Here again, these weak bands are too red-shifted to be assigned to progression from hot bands and the overall vibronic spectrum closely resembles the one record for $2-\mathrm{HPH}^{+}$with two sets of intense and weak vibronic transitions. 

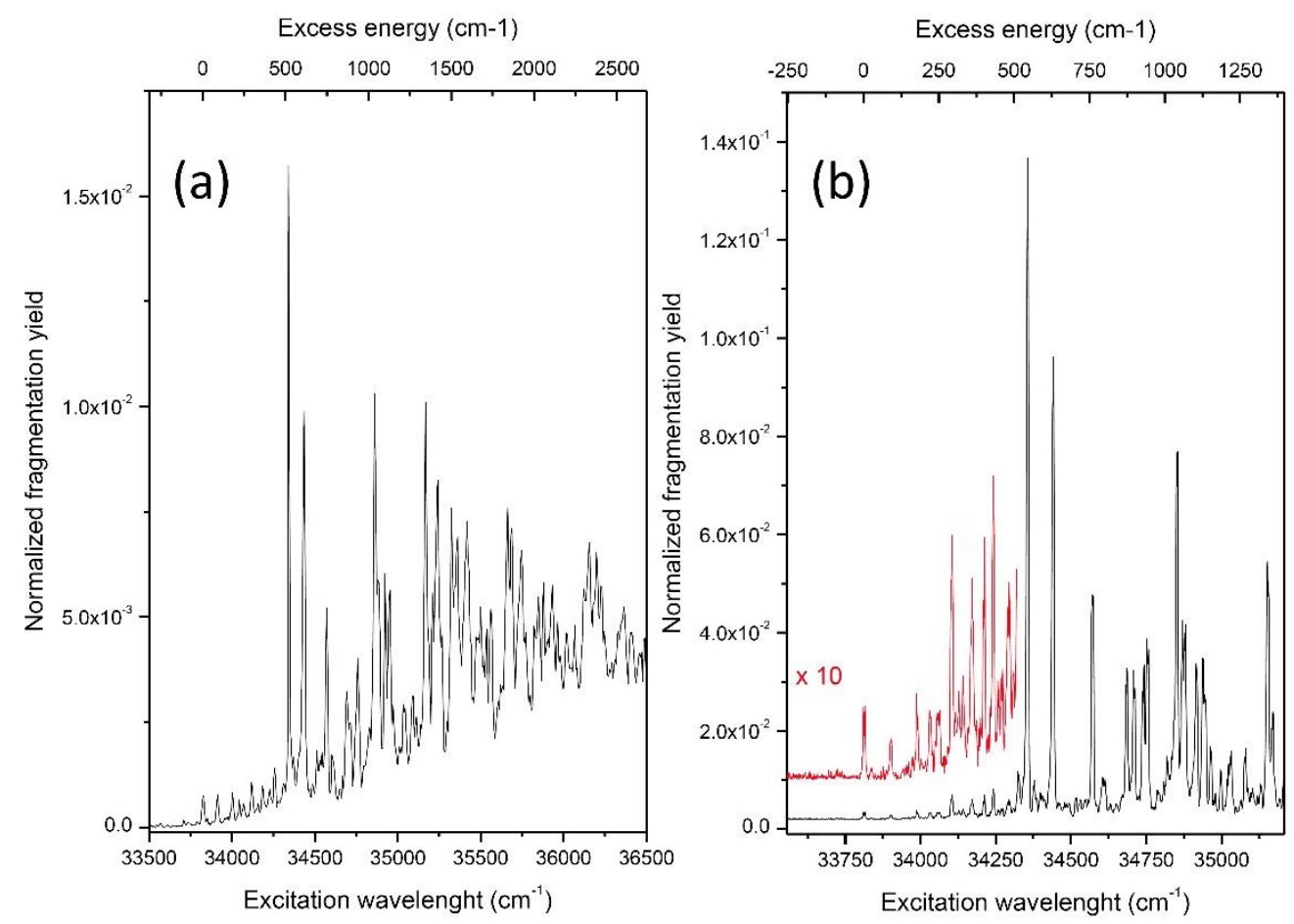

Figure 2. Photofragmentation spectrum of protonated $3-\mathrm{HPH}^{+}$recorded on the $\mathrm{m} / \mathrm{z} 68$ ionic fragment.(a) on the left, the spectrum is obtained over a large spectral range with the ps OPA laser ( $8 \mathrm{~cm}^{-1}$ resolution). (b) on the right, low frequency region obtained with the dye laser $\left(0.2 \mathrm{~cm}^{-1}\right.$ resolution).

For $3-\mathrm{HPH}^{+}$, two low-lying energy isomers (syn and anti) can be populated at low temperature. Ultraviolet-ultraviolet hole-burning spectroscopy was used to discriminate these conformational isomers. The very low intensity of the vibronic spectrum in the red of the first intense transition precludes to record any UV-UV HB signal in that spectral region. We thus turn to the region where intense transitions are detected. The burn laser was first set on the band at $34357 \mathrm{~cm}^{-1}$ (Fig. 3a). Most of the vibronic transitions are detected in the HB spectrum except a first one at $34686 \mathrm{~cm}^{-1}$. A second HB spectrum (Fig. 3b) is thus obtained by fixing the burn laser at this frequency. The two $\mathrm{HB}$ spectra allow recovering the entire vibronic spectrum of $3-\mathrm{HPH}^{+}$, which clearly reveals that only two conformers contribute to the experimental spectrum, at least in that spectral region. It should be noted that the burn laser seems to induce some hole-filling signals as reflected by few and weak positive HB bands for the two burn wavelengths. 

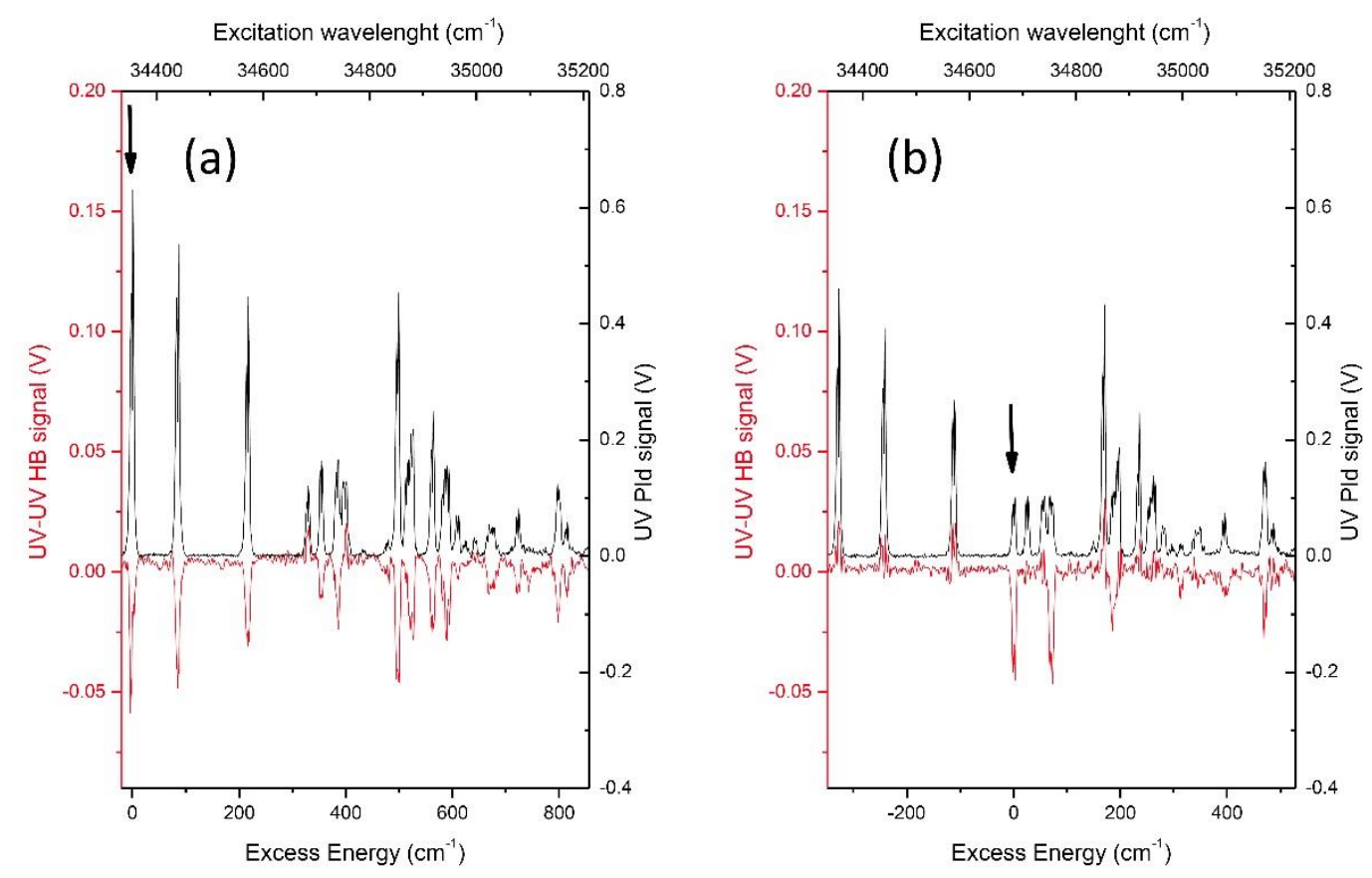

Figure 3. UV-UV hole-burning spectra of $3-\mathrm{HPH}^{+}$obtained at two burn laser frequencies (indicated by an arrow) of (a) $34357 \mathrm{~cm}^{-1}$ and (b) $34686 \mathrm{~cm}^{-1}$. For each plot (same vertical scale), the top trace (black line) is the UV photo-induced (PID) signal while the bottom trace (red line) is the UV-UV hole-burning (HB) signal.

\section{Protonated 4-Hydroxypyridine : 4- $\mathrm{HPH}^{+}$}

In that case, the fragmentation leads to three fragments: the main fragment is $\mathrm{m} / \mathrm{z} 68$ (CO loss) along with $\mathrm{m} / \mathrm{z} 78$ ( $\mathrm{H}_{2} \mathrm{O}$ loss) and a weak signal at $\mathrm{m} / \mathrm{z} 69$ ( $\mathrm{HCN}$ loss). The fragmentation kinetics is in the order of $2.5 \mathrm{~ms}$ which is much longer than for 2 - and $3-\mathrm{HPH}^{+}$. As for the other molecules, such long fragmentation kinetics strongly suggests that dissociation occurs in the ground electronic state after internal conversion. The photofragmentation spectrum of $4-\mathrm{HPH}^{+}$recorded with the ps OPA laser (Fig. 4a) is quite noisy and much less structured than the ones for 2 - and 3-HPH $\mathrm{H}^{+}$. With the dye laser, only a couple of sharp transitions are observed before an intense one followed by the onset of a broad continuum starting from roughly $40350 \mathrm{~cm}^{-1}$. The first transition is located at $39660 \mathrm{~cm}^{-}$ ${ }^{1}$ and should be assigned to the band origin. This $0_{0}{ }^{2}$ transition is significantly blue shifted as compared to those of protonated 2- and 3- hydroxypyridines. A first vibrational band is found 120 $\mathrm{cm}^{-1}$ above the origin transition while the most intense band appears $637 \mathrm{~cm}^{-1}$ higher in energy. As for the two other isomers, an intense vibronic transition is detected about $600 \mathrm{~cm}^{-1}$ above the first weak transition of the excitation spectrum but the overall vibronic spectrum is clearly different. 

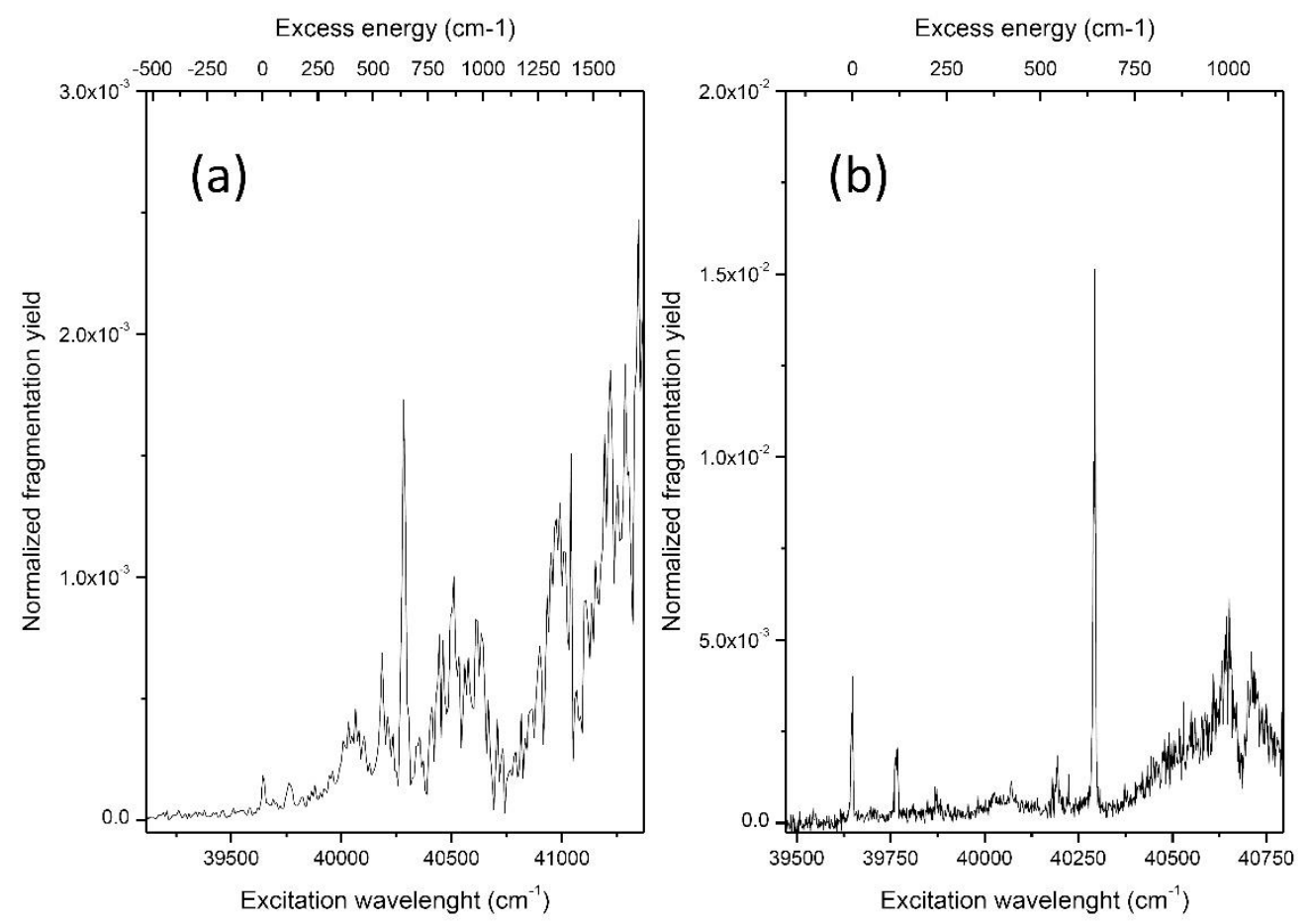

Figure 4. Photofragmentation spectrum of protonated $4-\mathrm{HPH}^{+}$averaged on the three ionic fragments at $\mathrm{m} / \mathrm{z}=78,69$ and 68. (a) on the left, the spectrum is obtained over a large spectral range with the ps OPA laser ( $8 \mathrm{~cm}^{-1}$ resolution). (b) on the right, low frequency region obtained with the dye laser $\left(0.2 \mathrm{~cm}^{-1}\right.$ resolution).

\section{4 - Discussion}

\section{Vertical and adiabatic excited state calculations}

$A b$ initio coupled-cluster (CC2 / aug-cc-pVDZ) calculations have been performed for the three $\mathrm{HPH}^{+}$ molecules. As expected, the syn conformer is more stable than the anti conformer by $4.15 \mathrm{kcal} / \mathrm{mol}$ and $0.95 \mathrm{kcal} / \mathrm{mol}$ for the $2-$ and $3-\mathrm{HPH}^{+}$, respectively. While the former one is thus not expected to be populated at low temperature, $3-\mathrm{HPH}^{+}$should adopt both conformations. Since the calculated electronic properties are similar and share the same trends for the syn and anti structures of 3$\mathrm{HPH}^{+}$, we will discuss only the results of the syn conformer. For all molecules, the ground state equilibrium structure is planar, all $\mathrm{HPH}^{+}$molecules thus belong to the $\mathrm{C}_{\mathrm{s}}$ point group. The vertical excited state energies of 2-3- and 4- $\mathrm{HPH}^{+}$are reported in Table 1 along with the corresponding frontier molecular orbital representation. The first low lying excited states are all of $\pi \pi^{*}$ character and no $n_{N} \pi^{*}$ is present since the lone pair of the pyridinic nitrogen is involved in bonding with the proton. The $\pi \sigma_{\mathrm{NH}}{ }^{*}$ state is calculated more than $2 \mathrm{eV}$ above the $\pi \pi^{*}$ state, which bears the oscillator strength for UV excitation, so that it is not involved in the deactivation process in $\mathrm{HPH}^{+}$. In $4-\mathrm{HPH}^{+}$, the first and second $\pi \pi^{*}$ states are almost degenerated and strongly mixed, with several orbitals involved in the frontier MO decomposition of the states. In these planar ground state structures, 
the lone pair of the hydroxyl group ( $p_{z}$ orbital) is in the same plane as the $\pi$ orbitals of the pyridine ring and contributes to the $\pi \pi^{*}$ transition.

Table 1. Electronic configurations and vertical transition energies (eV) of 2- 3- and 4-HPH ${ }^{+}$(CC2/aug-cc-pVDZ) syn conformers. In bold, $\pi \pi^{*}$ transition bearing the oscillator strength for UV excitation.

\begin{tabular}{|c|c|c|c|c|c|c|}
\hline & $2-\mathrm{HPH}^{+}\left(\mathrm{C}_{s}\right)$ & & $3-\mathrm{HPH}^{+}\left(\mathrm{C}_{s}\right)$ & & $4-\mathrm{HPH}^{+}\left(\mathrm{C}_{s}\right)$ & \\
\hline & Configuration & Energy & Configuration & Energy & Configuration & Energy \\
\hline $\mathrm{S}_{1}$ & $\begin{array}{c}\pi \pi^{*}\left(A^{\prime}\right) \\
\left(4 a^{\prime \prime}-5 a^{\prime \prime}\right)\end{array}$ & 4.82 & $\begin{array}{c}\pi \pi^{*}\left(A^{\prime}\right) \\
\left(4 a^{\prime \prime}-5 a^{\prime \prime}\right)\end{array}$ & 4.59 & $\begin{array}{c}\pi \pi^{*}\left(\mathrm{~A}^{\prime}\right) \\
\left(3 a^{\prime \prime}-6 a^{\prime \prime}\right)+\left(4 a^{\prime \prime}-5 a^{\prime \prime}\right)\end{array}$ & 5.43 \\
\hline $\mathrm{S}_{2}$ & $\begin{array}{c}\pi \pi *\left(A^{\prime}\right) \\
\left(4 a^{\prime \prime}-6 a^{\prime \prime}\right)\end{array}$ & 6.27 & $\begin{array}{c}\pi \pi *\left(\mathrm{~A}^{\prime}\right) \\
\left(4 a^{\prime \prime}-6 \mathrm{a}^{\prime \prime}\right)\end{array}$ & 5.75 & $\begin{array}{c}\pi \pi *\left(A^{\prime}\right) \\
\left(3 a^{\prime \prime}-5 a^{\prime \prime}\right)\end{array}$ & 5.58 \\
\hline $\mathrm{S}_{3}$ & $\begin{array}{c}\pi \pi *\left(A^{\prime}\right) \\
\left(3 a^{\prime \prime}-5 a^{\prime \prime}\right)\end{array}$ & 7.19 & $\begin{array}{c}\pi \pi *\left(A^{\prime}\right) \\
\left(3 a^{\prime \prime}-5 a^{\prime \prime}\right)\end{array}$ & 6.72 & $\begin{array}{c}\pi \pi *\left(\mathrm{~A}^{\prime}\right) \\
\left(4 \mathrm{a}^{\prime \prime}-5 \mathrm{a}^{\prime \prime}\right)+\left(3 \mathrm{a}^{\prime \prime}-6 \mathrm{a}^{\prime \prime}\right)\end{array}$ & 6.59 \\
\hline $\mathrm{S}_{4}$ & $\begin{array}{l}\pi \sigma_{N H} *\left(A^{\prime \prime}\right) \\
\left(4 a^{\prime \prime}-27 a^{\prime}\right)\end{array}$ & 7.28 & $\begin{array}{c}\pi \sigma_{N H} *\left(A^{\prime \prime}\right) \\
\left(4 a^{\prime \prime}-22 a^{\prime}\right)\end{array}$ & 7.07 & $\begin{array}{c}\pi \pi^{*}\left(A^{\prime}\right) \\
\left(4 a^{\prime \prime}-6 a^{\prime \prime}\right)\end{array}$ & 7.39 \\
\hline
\end{tabular}

Geometry optimizations of the first $\pi \pi^{*}$ state have been performed for the three $\mathrm{HPH}^{+}$molecules without symmetry constraint. In all cases, the optimization has converged to a planar $\pi \pi^{*}$ state preserving the Cs symmetry. However, these Cs structures are saddle points of the potential energy surface (PES) with one imaginary frequency of roughly $-50 /-80 \mathrm{~cm}^{-1}$ depending on the molecules. The associated mode corresponds to out-of-plane butterfly motion of the pyridinic ring. With such a low negative frequency in the $\pi \pi^{*}$ state, we cannot assert from the calculations whether or not the Cs structure is a saddle point or a true secondary minimum of the $\pi \pi^{*}$ PES.

In order to escape from the planar Cs geometry in $S_{1}$, the structures have been distorted along the calculated negative frequency mode. For $2-$ and $3-\mathrm{HPH}^{+}$, this has led to slightly non planar geometries in a boat-like, prefulvenic structure, the $\mathrm{N}_{1} \mathrm{H}$ and $\mathrm{C}_{4} \mathrm{H}$ groups pointing above the aromatic ring (See Fig. 5). These optimized structures are true minima of the PES without imaginary frequency. These two adiabatic structures closely resemble the $\mathrm{N}$-pyridinium excited state structure calculated at the DFT/CAM-B3LYP/aug-cc-pVDZ by Hansen et al. ${ }^{24}$, although the nitrogen pyramidalization is less pronounced in the present case. In pyridinium, the barrier height to planarity is calculated at $2000 \mathrm{~cm}^{-1}$, while in 2- and 3- $\mathrm{HPH}^{+}, \mathrm{CC} 2 /$ aug-cc-pVDZ calculations predict a very small barrier of about $100 \mathrm{~cm}^{-1}$ (see Table 2).

Such small energy difference, which more or less matches the amplitude of the negative frequency of the Cs structure, is certainly within the error of the CC2 method and the relative energy of the two structures in the excited state should be taken with great care. Experimentally, for 2- and 3$\mathrm{HPH}^{+}$, the first intense transition is blue-shifted by about $600 \mathrm{~cm}^{-1}$ as compared to the weak band 
origin. As it will be discussed below, the Franck-Condon analysis allows deciphering the contribution of two minima of the $\pi \pi^{*}$ PES in the experimental vibronic spectra of $2-$ and $3-\mathrm{HPH}^{+}$.

For 4- $\mathrm{HPH}^{+}$, geometry optimization following distortion along the butterfly mode has led to a conical intersection with the ground state $0.3 \mathrm{eV}$ below the energy of the Cs structure. At this point, the pyramidalization at the protonated nitrogen is large with the adjacent $\mathrm{CH}$ group pointing almost perpendicular to the pyridine cycle. The access, through a very small barrier, from the FranckCondon region (Cs structure) to the conical intersection in $4-\mathrm{HPH}^{+}$is consistent with the absence of a vibrational progression in the vibronic spectrum of $4-\mathrm{HPH}^{+}$.

$2-\mathrm{HPH}^{+}$
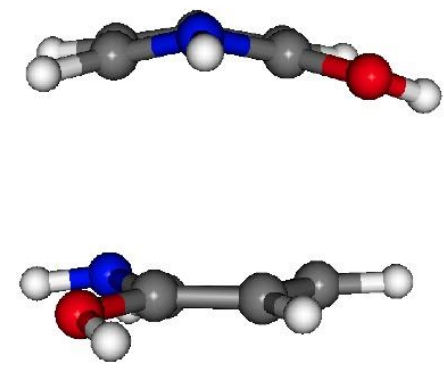

$3-\mathrm{HPH}^{+}$
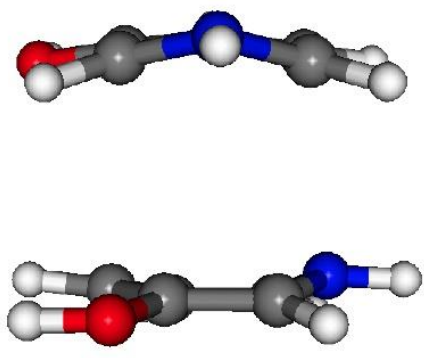

4- $\mathrm{HPH}^{+}$
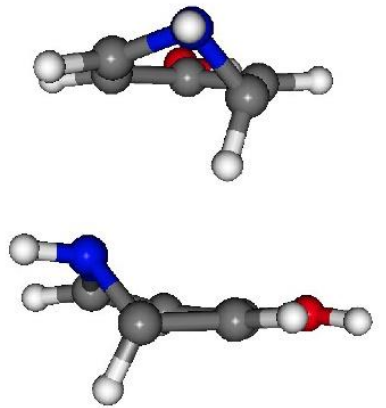

Figure 5. $\pi \pi^{*}$ excited state optimized prefulvenic structures of 2- and 3- $\mathrm{HPH}^{+}$. For $4-\mathrm{HPH}^{+}$, the geometry optimization leads to a conical intersection with the ground state, point where the optimization is stopped.

Table 2. Experimental and calculated $\pi \pi^{*}$ energy transition of 2-, 3- and 4- $\mathrm{HPH}^{+}$. Calculated energy transitions (CC2/augcc-pVDZ) corrected by the difference in zero point energy (ZPE) between the ground and excited states. All values are in $\mathrm{cm}^{-1}$.

\begin{tabular}{ccccc}
\hline molecules & Symmetry & $0_{0}{ }^{0}$ calc & $00^{0} \exp$ & $\Delta$ (calc-exp) \\
\hline \multirow{2}{*}{$2-\mathrm{HPH}^{+}$Syn } & prefulvenic & 35427 & 34806 & 621 \\
& Cs & 35522 & 35435 & 86 \\
$3-\mathrm{HPH}^{+}$Syn & prefulvenic & 34059 & 33814 & 245 \\
& Cs & 34124 & 34357 & -233 \\
$3-\mathrm{HPH}^{+}$Anti & prefulvenic & 34337 & - & \\
& Cs & 34481 & 34686 & -204 \\
$4-\mathrm{HPH}^{+}$ & prefulvenic & $\mathrm{IC}$ & 39660 & \\
& Cs & 40523 & 40297 & 226 \\
\hline
\end{tabular}




\section{Franck-Condon analysis}

The electronic spectrum of $4-\mathrm{HPH}^{+}$will not be discussed in this section because only a very few active vibronic bands are observed which precludes the assignment of the structure in the $\pi \pi^{*}$ PES. The vibronic spectra of 2- and 3- $\mathrm{HPH}^{+}$exhibit two sets of sharp transitions with quite different intensities. The excited state calculations have revealed the presence of two close lying structures in the $\pi \pi^{*}$ PES, an adiabatic prefulvenic structure and a second one of planar Cs geometry as in the electronic ground state. This is exactly what was found in protonated 2-aminopyiridine ${ }^{33}$. Its electronic spectrum has been assigned to the combination of two vibronic progressions of these two PES minima that have rather different Franck-Condon (FC) activities. Intense transitions were calculated for the Cs structure that correspond to the band origin (BO) along with the in-plane mode $\left(a^{\prime}\right)$ of the pyridinic ring, while the red-shifted low intensity bands are assigned to the adiabatic minimum with progression of out-of-plane modes. In 2 - and $3-\mathrm{HPH}^{+}$, the simulated vibronic spectra of the adiabatic minima have been calculated and their overall intensity are ten-fold weaker than those for the Cs structures. For $3-\mathrm{HPH}^{+}$, the presence of the two conformers and the impossibility to perform the hole-burning spectroscopy in the low frequency region makes the assignment highly hypothetical. Comparison between the calculated and experimental spectra are thus reported in Fig. 6 for $2-\mathrm{HPH}^{+}$. The calculated band origin has a very weak intensity. A progression of the butterfly mode $v_{1}\left(a^{\prime \prime}\right)$ calculated at $90 \mathrm{~cm}^{-1}$ built from the BO with a maximum at 3-4 quanta closely matches the experimental low frequency progression of $82 \mathrm{~cm}^{-1}$ experimentally observed for $2-\mathrm{HPH}^{+}$. The same $v_{1}$ progression built from the Franck-Condon active modes $v_{4}, v_{5}, v_{7}, v_{13}$ and $v_{15}$ is calculated. If the intensities of the calculated $0_{0}{ }^{0}$ and $v_{1}$ progression are scaled to the corresponding experimental bands, it is clear from these simulations that the intense experimental transitions from $630 \mathrm{~cm}^{-1}$ above the $\mathrm{BO}$ cannot be attributed to the adiabatic minimum. 


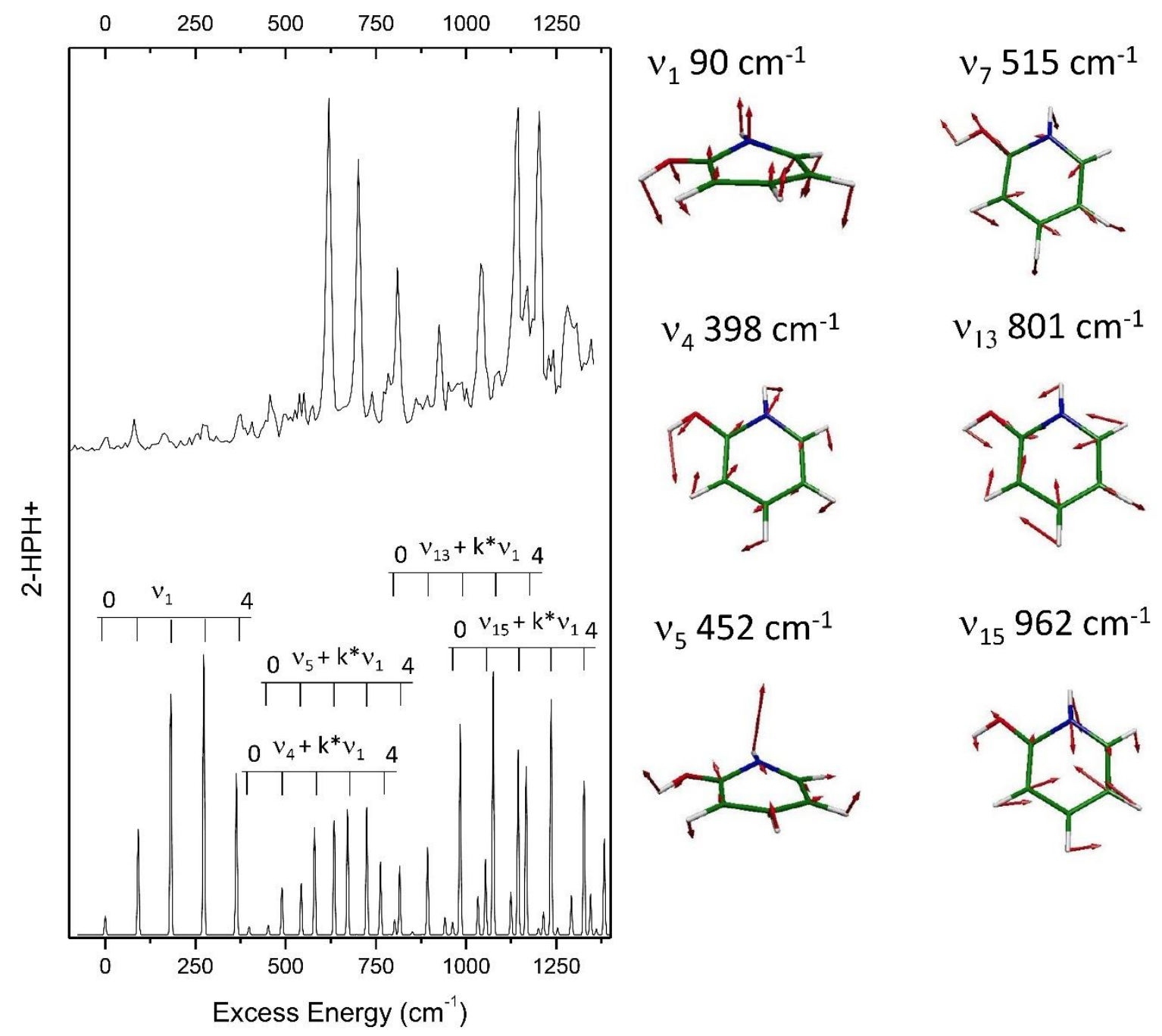

Figure 6. Comparison of the experimental (top) and Franck-Condon simulated spectrum of the prefulvenic structure of $2-\mathrm{HPH}^{+}$.

In Figure 7, the experimental vibronic spectrum of $2-\mathrm{HPH}^{+}$is compared to the Franck-Condon simulations of the Cs structure (Fig. 7a) with its origin set at the most intense vibronic band (35 435 $\mathrm{cm}^{-1}$ ) of the experimental spectrum. It is noteworthy that the calculated and experimental band origins (Table 2) of the Cs structure only differ by less than $100 \mathrm{~cm}^{-1}$. Besides, their calculated transition intensities are one order of magnitude larger than the ones of the prefulvenic structure. Selection rules of the Cs point group impose that only totally symmetric a' vibrational modes are active along with even quanta of out-of-plane a" modes. This is exactly reproduced in the simulated FC spectrum. The in-plane active modes of the Cs structure are calculated at 402, 503, 547, 804 and $958 \mathrm{~cm}^{-1}$ and correspond to $\mathrm{COH}$ bending, $6 \mathrm{a}-\mathrm{b}$, mode 1 and $12 \mathrm{~b}$ according to the Wilson notation, respectively (see Fig. 7). Only the first a" mode $\left(v_{1}=162 \mathrm{~cm}^{-1}\right)$ with two quanta has non negligible intensity in the calculated FC spectrum. While these active modes nicely match some of the intense experimental vibronic transitions, others are obviously missing in this simulation, in particular, the 
two first transitions found at +76 and $+183 \mathrm{~cm}^{-1}$ from the $35435 \mathrm{~cm}^{-1}$ band. The absence of the active low frequency modes of the Cs structure suggests that the missing bands might come from vibronic coupling between the prefulvenic and Cs minima.

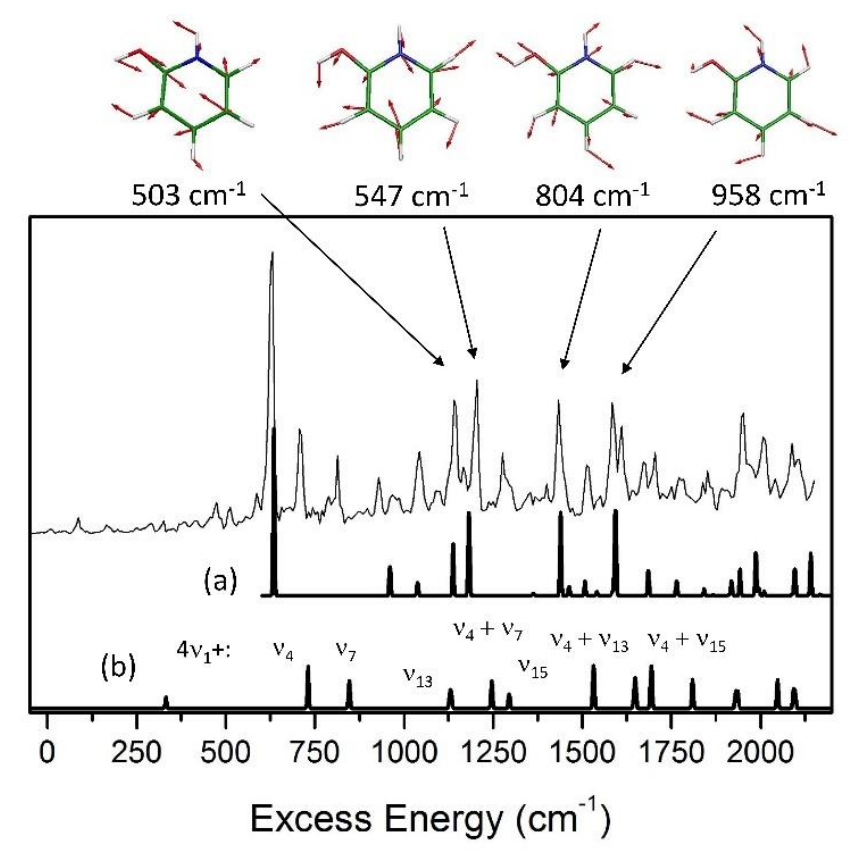

Figure 7. Comparison of the $2-\mathrm{HPH}^{+}$experimental (top) and calculated Franck-Condon spectra of (a) Cs structure and (b) the prefuvenic structure obtained as a combination of 4 quanta of the butterfly mode $v_{1}$ with the in-plane (a') modes. All spectra are plotted as a function of excess energy $\left(\mathrm{cm}^{-1}\right)$ from the band origin of the prefulvenic structure. The inplane $\left(a^{\prime}\right)$ modes are reported for the Cs structure.
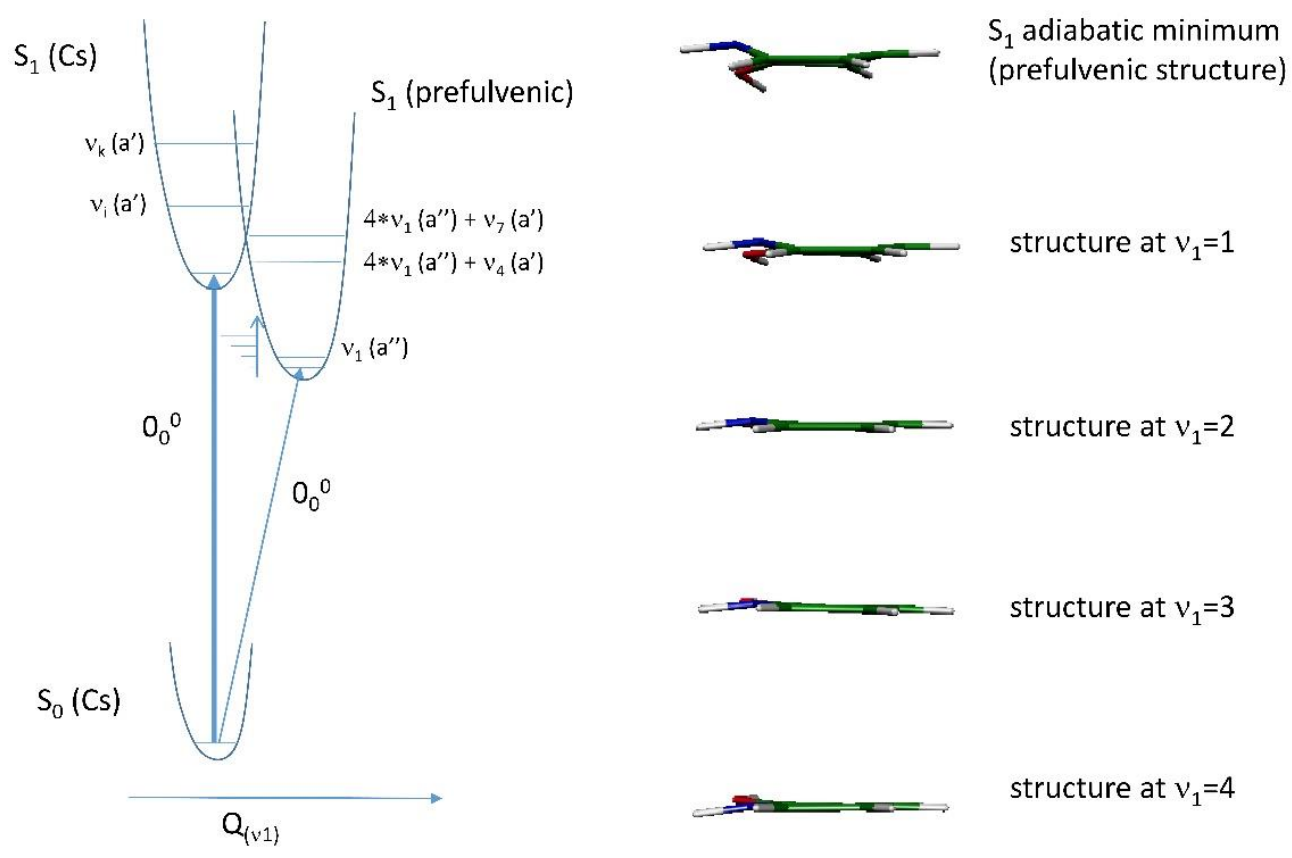

$\mathrm{S}_{1}$ adiabatic minimum

structure at $v_{1}=1$

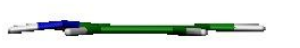

structure at $v_{1}=2$

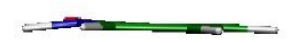

structure at $v_{1}=3$

structure at $v_{1}=4$

Figure 8. (left) Schematic diabatic representation of the double-well potential with $\mathrm{Q}_{(v 1)}$ as the butterfly bending mode coordinate that connects the planar $\left(\mathrm{C}_{s}\right)$ and prefulvenic minima in $\mathrm{S}_{1}$. (Right) distorted structures of 2- $\mathrm{HPH}^{+}$as a function of butterfly mode $v_{1}$ progression. 
Figure 8 shows a schematic diabatic representation of the double-well potential (harmonic approximation) with $Q_{(v 1)}$ as the butterfly bending mode coordinate relevant for this spectroscopic study. Vibronic transitions from the Cs ground state structure to the Cs excited state structure have large intensities for the in-plane ( $\left.a^{\prime}\right)$ modes, while Franck-Condon active transitions to the prefulvenic excited state structure involve a progression of the butterfly $v_{1}$ mode. We have used the screwer module in Turbomole to distort the $2-\mathrm{HPH}^{+}$molecule along the normal mode $v_{1}$ as a function of the temperature. Within the harmonic oscillator approximation, the energy of a vibrational normal mode $i\left(E_{i}\right)$ is given by $E_{i}=1 / 2 \omega_{i}{ }^{2} * Q_{i}{ }^{2}$, where $\omega_{i}$ and $Q_{i}$ are the wavenumber and normal coordinate of mode $i$, respectively. If one substitutes $E$ by $k_{b} T$, ( $k_{b}$ for Boltzmann constant and $T$ the temperature), the coordinates can be distorted along the butterfly mode $\left(v_{1}=90 \mathrm{~cm}^{-1}\right.$ for 2- $\mathrm{HPH}^{+}$) with a temperature step of $125 \mathrm{~K}$. In the right panel of Fig. 8, the corresponding distorted structures of $2-\mathrm{HPH}^{+}$are reported, and it can be readily checked that with 3 or 4 quanta in $v_{1}$, the prefulvenic structure has almost recovered the planarity. Indeed, the calculated FC spectrum of the prefulvenic structure (Fig. 6) reveals that the largest transitions are those with 3 quanta in $\mathrm{v}_{1}$, which scales the prefulvenic to planar coordinate displacement in the excited state. Qualitatively, a vibronic coupling between the prefulvenic and planar (Cs) minima should involve vibrations of the prefulvenic state that recover the planar symmetry (butterfly mode) and match the general selection rules of the Cs point group, i.e. totally symmetric a' modes. The simulated FC spectrum of Fig. $7 \mathrm{~b}$ has been calculated as combination bands of 4 quanta in the butterfly mode and all the inplane bending and stretching modes $\left(a^{\prime}\right)$ of the adiabatic structure. Most of the missing transitions in the simulated spectrum of the Cs structure, in particular the two intense transition at $+76 \mathrm{~cm}^{-1}$ and $+183 \mathrm{~cm}^{-1}$ from the $\mathrm{BO}$ of the Cs structure, can now be assigned to the contribution of combination bands of the prefulvenic structure with in-plane $\left(a^{\prime}\right)$ modes and 4 quanta of the butterfly mode needed to recover the planarity. This diabatic harmonic formalism used to simulate the experimental spectrum is certainly a crude approximation of the true adiabatic potential energy surface of the excited state. Nevertheless, it provides a very good qualitative and satisfying quantitative agreement with the experimental spectrum.

The same procedure has been applied to $3-\mathrm{HPH}^{+}$to assign the intense vibronic bands of the experimental spectrum. As already pointed out, two conformers account for all the intense vibronic transitions. The band origins of the syn and anti conformers in the planar Cs structure have been assigned through the hole-burning spectroscopy and compared to the calculated ones. The syn 
conformer has its $\mathrm{BO}$ at $34357 \mathrm{~cm}^{-1}, 329 \mathrm{~cm}^{-1}$ to the red of the $\mathrm{BO}$ of the anti conformer. Both the absolute and relative adiabatic transition energies are very well reproduced by the calculation, in particular the splitting between the two conformers predicted at $357 \mathrm{~cm}^{-1}$, i. e. within $30 \mathrm{~cm}^{-1}$ of the experimental one (see Table 2). The UV-UV hole-burning spectrum of the anti conformer (Fig. 3b) covers a limited spectral range of $500 \mathrm{~cm}-1$ where only the first two in-plane active modes ( $a^{\prime}$ ) of the Cs structure are detected at 388 and $500 \mathrm{~cm}-1$ along with combination bands involving 4 quanta of the mode $v_{1}$ of the adiabatic minimum. In the Cs structure, the frequencies of in plane modes (breathing vibration of the cycle) are not quite different for the three $\mathrm{HPH}+$, even less for the syn/anti conformers of $3 \mathrm{HPH}^{+}$. Nevertheless, as for $2-\mathrm{HPH}^{+}$, the intense vibronic bands assigned to the syn conformer in Figure 3a can be reproduced with the active in-plane a' modes of the Cs minimum along with combination of 4 quanta of the butterfly motion with in-plane a' modes of the prefulvenic minimum structure (Fig. SI1).

\section{5 - Conclusions}

We have investigated the photofragmentation processes in protonated hydroxypyridine through a combined experimental and theoretical study. Although protonated ortho, meta and para hydroxypyridine appear as simple model molecules that mimic the molecular subunits comprising larger biologically relevant compounds, the understanding of their vibronic spectroscopy is more complex than expected. These three molecules show well resolved electronic excitation spectra, with a strong influence of the position of the hydroxyl group. In particular, the band origin of 4-HPH is significantly blue-shifted in comparison with 2- and 3-HPH $\mathrm{HPV}^{+}$-UV hole burning spectroscopy for the $3-\mathrm{HPH}^{+}$clearly reveals the presence of two syn- and anti-conformers at low temperature. For all molecules, the weak intensity of the band origin along with the progression on low frequency modes reveals a geometry change in the excited state, as predicted by the prefulvenic structure found through excited-state optimized geometry calculated at the CC2 / aug-cc-pVDZ. Besides, the intense vibronic transitions are assigned to the planar Cs structure that corresponds to tenfold larger intensities. For both adiabatic and Cs structures, the calculated band origins including ZPE corrections are in good agreement with the experimental data within an absolute error of about $500 \mathrm{~cm}^{-1}$. Finally, a strong vibronic interaction between the prefulvenic and planar structures in the excited state seems to account for the experimental spectra. 


\section{Acknowledgments}

We acknowledge the use of the computing facility clusters GMPCS of the LUMAT federation (FRLUMAT 2764) and MAGI of the University Paris 13.

\section{References}

(1) G.G. Gomez-Tejedor, M.C. Fuss, Radiation Damage in Biomolecular Systems, Springer, 2012.

(2) D. Picconi, F.J.A. Ferrer, R. Improta, A. Lami, F. Santoro, Quantum-classical effective-modes dynamics of the $\pi \pi^{*} \rightarrow \mathrm{n} \pi^{*}$ decay in $9 \mathrm{H}$-adenine. A quadratic vibronic coupling model, Faraday Discuss., 2013, 163, 223-242.

(3) A.L. Sobolewski, W. Domcke, C. Dedonder-Lardeux, C. Jouvet, Excited-state hydrogen detachment and hydrogen transfer driven by repulsive ${ }^{1} \pi \sigma^{*}$ states: A new paradigm for nonradiative decay in aromatic biomolecules, Phys. Chem. Chem. Phys., 2002, 4, 1093-1100.

(4) G.M. Roberts, C.A. Williams, H. Yu, A.S. Chatterley, J.D. Young, S. Ullrich, V.G. Stavros, Probing ultrafast dynamics in photoexcited pyrrole : timescales for $\pi \sigma^{*}$ mediated $\mathrm{H}$-atom elimination, Faraday Discuss., 2013, 163, 95-116.

(5) M.N.R. Ashfold, B. Cronin, A.L. Devine, R.N. Dixon, M.G.D. Nix, The Role of $\pi \sigma^{*}$ Excited States in the Photodissociation of Heteroaromatic Molecules, Science, 2006, 312, 1637-1640.

(6) C.Y. Switzer, S.E. Moroney, S.A. Benner, Enzymatic recognition of the base pair between isocytidine and isoguanosine, Biochemistry, 1993, 32, 10489-10496.

(7) D. Lee, S.J. Baek, K.-W. Choi, Y.S. Choi, S.K. Kim, Resonantly-enhanced Two-photon Ionization and Mass-Analysed Threshold Ionization (MATI) Spectroscopy of 2-Hydroxypyridine, Bull. Korean Chem. Soc. 2002, 23 (2), 277-280.

(8) K. Sakota, S. Tokuhara, H. Sekiya, Dispersed fluorescence spectroscopy of 2-hydroxypyridine and its cyclically hydrogen-bonded water clusters in the gas phase : an examination of occurrence of excited-state proton transfer, Chem. Phys. Lett., 2007, 448, 159-163.

(9) M.R. Nimlos, D.F. Kelley, E.R. Bernstein, Spectroscopy, structure, and proton dynamics of 2hydroxypyridine and its clusters with water and ammonia, J. Phys. Chem., 1989, 93 (2), 643-651.

(10) Y. Matsuda, T. Ebata, N. Mikami, IR-UV Double-Resonance Spectroscopic Study of 2Hydroxypyridine and Its Hydrogen-Bonded Clusters in Supersonic Jets, J. Phys. Chem. A, 2001, 105, 3475-3480. 
(11) M. Meuwly, A. Müller, S. Leutwyler, Energetics, dynamics and infrared spectra of the DNA basepair analogue 2-pyridone 2-hydroxypyridine, Phys. Chem. Chem. Phys., 2003, 5, 2663-2672.

(12) G.T. Wondrak, M.J. Roberts, D. Cervantes-Laurean, M.K. Jacobson, E.L. Jacobson, Proteins of the Extracellular Matrix Are Sensitizers of Photo-oxidative Stress in Human Skin Cells, J. Invst. Dermatol., 2003, 121, 578-586.

(13) G.T. Wondrak, M.J. Roberts, M.K. Jacobson, E.L. Jacobson, 3-Hydroxypyridine Chromophores Are Endogenous Sensitizers of Photooxidative Stress in Human Skin Cells, The Journal of Biological Chemistry, 2004, 279 (29), 30009-30020.

(14) F.O. Talbot, T. Tabarin, R. Antoine, M. Broyer, P. Dugourd, Photodissociation Spectroscopy of Trapped Protonated Tryptophan, J. Chem. Phys., 2005, 122, 074310.

(15) O.V. Boyarkin, S.R. Mercier, A. Kamariotis, T.R. Rizzo, Electronic Spectroscopy of Cold Protonated Tryptophan and Tyrosine, J. Am. Chem. Soc., 2006, 128, 2816-2817.

(16) J.A. Stearns, S.R. Mercier, C. Seaiby, M. Guidi, O.V. Boyarkin, T.R. Rizzo, Conformation-Specific Spectroscopy and Photodissociation of Cold, Protonated Tyrosine and Phenylalanine, J. Am. Chem. Soc., 2007, 129, 11814-11820.

(17) D. Nolting, C. Marian, R. Weinkauf, Protonation effect on the electronic spectrum of tryptophan in the gas phase, Phys. Chem. Chem. Phys., 2004, 6, 2633-2640.

(18) M. Berdakin, G. Féraud, C. Dedonder-Lardeux, C. Jouvet, G.A. Pino, Excited States of Protonated DNA/RNA bases, Phys. Chem. Chem. Phys., 2014, 16, 10643-10650.

(19) S. Ф. Pedersen, C.S. Byskov, F. Turecek, S.B. Nielsen, Structures of Protonated Thymine and Uracil and Their Monohydrated Gas-Phase Ions from Ultraviolet Action Spectroscopy and Theory, J. Phys. Chem. A, 2014, 118 (24), 4256-4265.

(20) S.B. Nielsen, J. Wyer, Physical Chemistry in Action : Photophysics of Ionic Biochromophores, Springer-Verlag, Berlin Heidelberg, 2013.

(21) G. Féraud, M. Berdakin, C. Dedonder, C. Jouvet, G.A. Pino, Excited States of Proton-bound Base Homo-dimers : Pyrimidines, J. Phys. Chem. B, 2015, 119 (6), 2219-2228.

(22) Y.S. Wang, C.H. Tsai, Y.T. Lee, H.C. Chang, J.C. Jiang, O. Asvany, S. Schlemmer, D. Gerlich, Investigations of Protonated and Deprotonated Water Clusters Using a Low-Temperature 22-Pole Ion Trap, J. Phys. Chem. A, 2003, 107, 4217-4225.

(23) N. Esteves-López, C. Dedonder-Lardeux, C. Jouvet, Excited State of protonated benzene and toluene, J. Chem. Phys., 2015, 143, 074303.

(24) C.S. Hansen, S.J. Blanksby, N. Chalyavi, E.J. Bieske, J.R. Reimers, A.J. Trevitt, Ultraviolet photodissociation action spectroscopy of the N-pyridinium cation, J. Chem. Phys., 2015, 142, 014301. 
(25) M.F. Rode, A.L. Sobolewski, C. Dedonder, C. Jouvet, O. Dopfer, Computational Study on the Photophysics of Protonated Benzene, J. Phys. Chem. A, 2009, 113, 5865-5873.

(26) M. Rosenberg, C. Dahlstrand, K. Kilsa, H. Ottoson, Excited State Aromaticity and Antiaromaticity : Opportunities for Photophysical and Photochemical Rationalizations, Chem. Rev., 2014, 114, 5379-5425.

(27) I. Palmer, I. Ragazos, F. Bernadi, M. Olivucci, M. Robb, An MC-SCF study of the S1 and S2 photochemical reactions of benzene, J. Am. Chem. Soc., 1993, 115 (2), 673-682.

(28) A. Sobolewski, C. Woywod, W. Domcke, $A b$ initio investigation of potential-energy surfaces involved in the photophysics of benzene and pyrazine, J. Chem. Phys., 1993, 98 (7), 5627-5641.

(29) M. Chachisvilis, A. Zewail, Femtosecond Dynamics of Pyridine in the Condensed Phase : Valence Isomerization by Conical Intersections, J. Phys. Chem. A, 1999, 103 (37), 7408-7418.

(30) D. Zhong, W.G. Diau, T.M. Bernhardt, S.D. Feyter, J.D. Roberts, A.H. Zewail, Femtosecond dynamics of valence-bond isomers of azines : transition states and conical intersections, Chem. Phys. Lett., 1998, 298, 129-140.

(31) A. Sobolewski, W. Domcke, Photophysically relevant potential energy functions of low-lying singlet states of benzene, pyridine and pyrazine : an ab initio study, Chem. Phys. Lett., 1991, 180, 381-386.

(32) K. Grove, R.A. King, U. Burger, Photo-rearrangement of N-substituted pyridinium and metaalkoxypyridinium ions, J. Mol. Struct. : THEOCHEM, 2007, 807, 25-32.

(33) M. Broquier, S. Soorkia, C. Dedonder-Lardeux, C. Jouvet, P. Theulé, G. Grégoire, Twisted Intramolecular Charge Transfer in Protonated Amino Pyridine, J. Phys. Chem. A, 2016, 120 (21), 3797-3809.

(34) S. Soorkia, M. Broquier, G. Grégoire, Multiscale excited state lifetimes of protonated dimethyl aminopyridines, Phys.Chem.Chem.Phys., 2016, 18, 23785-23794.

(35) X.B. Wang, L.S. Wang, Development of a Low-Temperature Photoelectron Spectroscopy Instrument Using an Electrospray lon Source and a Cryogenically Controlled Ion Trap, Rev. Sci. Instrum., 2008, 79, 073108.

(36) G. Féraud, M. Broquier, C. Dedonder, C. Jouvet, G. Grégoire, S. Soorkia, Excited State Dynamics of Protonated Phenylalanine and Tyrosine: Photo-Induced Reactions Following Electronic Excitation, J. Phys. Chem. A, 2015, 119 (23), 5914-5924.

(37) M. Broquier, S. Soorkia, G. Grégoire, A comprehensive study of cold protonated tyramine: UV photodissociation experiments and ab initio calculations, Phys.Chem.Chem.Phys., 2015, 17, 2585425862.

(38) H. Kang, G. Feraud, C. Dedonder-Lardeux, C. Jouvet, New Method for Double-Resonance Spectroscopy in a Cold Quadrupole Ion Trap and Its Application to UV-UV Hole-Burning Spectroscopy of Protonated Adenine Dimer, J. Phys. Chem. Lett., 2014, 5 (18), 2760-2764. 
(39) T.S. Zwier, The Spectroscopy of Solvation in Hydrogen-bonded Aromatic Clusters, Annu. Rev. Phys. Chem., 1996, 47, 205-241;

(40) A. Sen, V. Lepere, K. Le Barbu-Debus, A. Zehnacker, How do Pseudoenantiomers Structurally Differ in the Gas Phase? An IR/UV Spectroscopy Study of Jet-Cooled Hydroquinine and Hydroquinidine, ChemPhysChem, 2013, 14 (15), 3559-3568.

(41) R. Ahlrichs, M. Bär, M. Häser, H. Horn, C. Kölmel, Electronic Structure Calculations on Workstation Computers : The Program System Turbomole, Chem. Phys. Lett., 1989, 162, 165-169.

(42) F. Weigend, M. Häser, RI-MP2 : First Derivatives and Global Consistency, Theor. Chem. Acc., 1997, 97, 331-340.

(43) T.H. Dunning, Gaussian Basis Sets for Use in Correlated Molecular Calculations. I. The Atoms Boron through Neon and Hydrogen, J. Chem. Phys., 1989, 90, 1007.

(44) C.M. Western, PGOPHER, a Program for Simulating Rotational Structure, University of Bristol : Bristol, U.K., 2010 ; available at http://pgopher.chm.bris.ac.uk. 


\section{Supplementary Materials:}

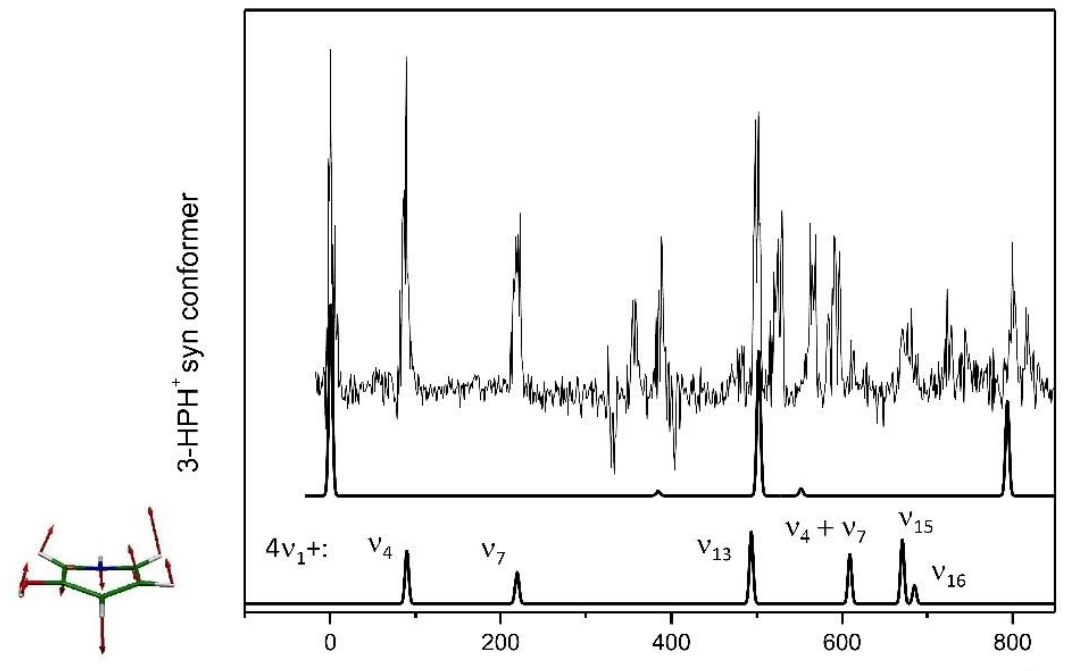

$v_{1} 95 \mathrm{~cm}^{-1}$

Excess Energy above $\mathrm{BO}$ of Cs structure, syn conformer $\left(\mathrm{cm}^{-1}\right)$
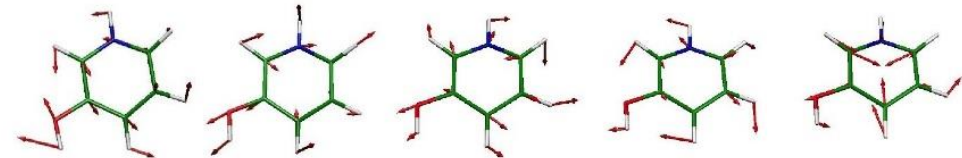

$v_{4} 390 \mathrm{~cm}^{-1} \quad v_{7} 519 \mathrm{~cm}^{-1} \quad v_{13} 793 \mathrm{~cm}^{-1} \quad v_{15} 971 \mathrm{~cm}^{-1} \quad v_{16} 985 \mathrm{~cm}^{-1}$

Figure SI1: Comparison of the experimental (top) and Franck-Condon simulated spectra of the 3- $\mathrm{HPH}^{+}$syn conformer. 